\title{
Decay Widths of Excited Heavy Mesons From QCD Sum Rules at the Leading Order of HQET
}

\author{
Yuan-Ben Dai ${ }^{a}$, Chao-Shang Huang ${ }^{a}$, Ming-Qiu Huang ${ }^{b, c},{\text { Hong-Ying } \operatorname{Jin}^{c}, \text { Chun Liu }}^{d}$ \\ ${ }^{a}$ Institute of Theoretical Physics, Academia Sinica, P.O.Box 2735, Beijing 100080, China \\ ${ }^{b}$ CCAST (World Laboratory) P.O. Box 8730, Beijing, 100080 \\ ${ }^{c}$ Institute of High Energy Physics, Academia Sinica, P.O.Box 918, Beijing 100039, China \\ ${ }^{d}$ Center For Theoretical Physics, Seoul National University, Seoul, 151-742, Korea
}

\begin{abstract}
The widths for pionic decays of lowest two excited doublets $\left(0^{+}, 1^{+}\right)$and $\left(1^{+}, 2^{+}\right)$of heavy mesons are studied with QCD sum rules in the leading order of heavy quark effective theory. The ambiguity due to presence of two distinct $1^{+}$states are solved. The soft pion approximation is not used.

PACS number(s): 13.25.Ft, 12.39.Hg, 11.55.Hx, 12.38.Lg
\end{abstract}

Typeset using REVTEX 


\section{INTRODUCTION}

The properties of excited heavy mesons have attracted attention in recent years. The mass and decay widths have been studied with potential model combining with data for $K$ mesons in [1], with relativistic Bethe-Salpeter equation in [2,3] and with QCD sum rules in [4]. In work [5] the masses of low lying excited heavy mesons $\left(2^{+}, 1^{+}\right)$and $\left(1^{+}, 0^{+}\right)$were studied with QCD sum rules in the heavy quark effective theory (HQET) [6] up to the order of $\mathcal{O}\left(1 / m_{Q}\right)$, where $m_{Q}$ is the heavy quark mass. The present work is devoted to the decay widths of these mesons in the same formalism.

One problem encountered in studying the decay widths of excited heavy mesons with QCD sum rules is the following. Except for the lowest states $0^{-}, 1^{-}$, the spectra contains a pair of states for any spin-parity $j^{P}$ with close values in their masses but quite different in magnitudes of their decay widths. In our case, one of the two $1^{+}$states is a narrow resonance decaying mainly by emitting a $D$ wave pion, while the other one is a very wide resonance decaying by emitting a $S$ wave pion. An interpolating current used for the narrow $1^{+}$state with a small coupling to the other $1^{+}$state may cause sizable error in the result of calculation. It is only in the $m_{Q} \rightarrow \infty$ limit, there is a conserved quantum number $j_{\ell}$, the angular momentum of the light component, which can be used to differentiate the two states. Therefore, HQET has important advantage for this purpose.

The proper interpolating current $J_{j, P, j_{\ell}}^{\alpha_{1} \cdots \alpha_{j}}$ for the states with the quantum number $j, P$, $j_{\ell}$ in HQET was given in [5]. They were proved to satisfy the following conditions

$$
\begin{aligned}
\left\langle 0\left|J_{j, P, j_{\ell}}^{\alpha_{1} \cdots \alpha_{j}}(0)\right| j^{\prime}, P^{\prime}, j_{\ell}^{\prime}\right\rangle= & f_{P j_{l}} \delta_{j j^{\prime}} \delta_{P P^{\prime}} \delta_{j \ell} j_{\ell}^{\prime} \eta^{\alpha_{1} \cdots \alpha_{j}} \\
i\left\langle 0\left|T\left(J_{j, P, j_{\ell}}^{\alpha_{1} \cdots \alpha_{j}}(x) J_{j^{\prime}, P^{\prime}, j_{\ell}^{\prime}}^{\beta_{1} \cdots \beta_{j^{\prime}}}(0)\right)\right| 0\right\rangle= & \delta_{j j^{\prime}} \delta_{P P^{\prime}} \delta_{j \ell j_{\ell}}(-1)^{j} \mathcal{S} g_{t}^{\alpha_{1} \beta_{1}} \cdots g_{t}^{\alpha_{j} \beta_{j}} \\
& \times \int d t \delta(x-v t) \Pi_{P, j_{\ell}}(x)
\end{aligned}
$$

in the $m_{Q} \rightarrow \infty$ limit, where $\eta^{\alpha_{1} \cdots \alpha_{j}}$ is the polarization tensor for the spin $j$ state, $v$ is the velocity of the heavy quark, $g_{t}^{\alpha \beta}=g^{\alpha \beta}-v^{\alpha} v^{\beta}$ is the transverse metric tensor, $\mathcal{S}$ denotes symmetrizing the indices and subtracting the trace terms separately in the sets 
$\left(\alpha_{1} \cdots \alpha_{j}\right)$ and $\left(\beta_{1} \cdots \beta_{j}\right), f_{P, j_{\ell}}$ and $\Pi_{P, j_{\ell}}$ are a constant and a function of $x$ respectively which depend only on $P$ and $j_{\ell}$. Because of equations (1) and (2), the sum rule in HQET for decay widths derived from a correlator containing such currents receive no contribution from the unwanted states with the same spin-parity as the states under consideration in the $m_{Q} \rightarrow \infty$. Starting from the calculations in the leading order, the decay amplitudes for finite $m_{Q}$ can be calculated unambiguously order by order in the $1 / m_{Q}$ expansion in HQET.

\section{SUM RULES FOR DECAY AMPLITUDES}

In the present work we shall confine ourselves to the lowest lying excited states in the leading order of $1 / m_{Q}$ expansion. Denote the doublet $\left(1^{+}, 2^{+}\right)$with $j_{\ell}=3 / 2$ by $\left(B_{1}, B_{2}^{*}\right)$ and the doublet $\left(0^{+}, 1^{+}\right)$with $j_{\ell}=1 / 2$ by $\left(B_{0}^{\prime}, B_{1}^{\prime}\right)$. From covariance and conservation of the angular momentum of the light component in the $m_{Q} \rightarrow \infty$ limit, the amplitudes for the decay of $B_{1}, B_{2}^{*}$ to the ground states $B, B^{*}$ in the doublet $\left(0^{-}, 1^{-}\right)$with $j_{\ell}=1 / 2$ have the following forms

$$
\begin{aligned}
M\left(B_{1} \rightarrow B^{*} \pi\right) & =I \epsilon_{\mu}^{*} \eta_{\nu}\left(q_{t}^{\mu} q_{t}^{\nu}-\frac{1}{3} g_{t}^{\mu \nu} q_{t}^{2}\right) g\left(B_{1}, B^{*}\right), \\
M\left(B_{2}^{*} \rightarrow B \pi\right) & =I \eta_{\mu \nu} q_{t}^{\mu} q_{t}^{\nu} g\left(B_{2}^{*}, B\right), \\
M\left(B_{2}^{*} \rightarrow B^{*} \pi\right) & =I i \varepsilon_{\alpha \beta \sigma \rho} \epsilon^{* \alpha} v^{\beta} \eta^{\sigma \mu} q_{t}^{\rho} q_{t \mu} g\left(B_{2}^{*}, B^{*}\right),
\end{aligned}
$$

where $\eta_{\mu \nu}, \eta_{\mu}$ and $\epsilon_{\mu}$ are polarization tensors for states $2^{+}, 1^{+}$and $1^{-}$respectively. $q_{t \mu}=$

$q_{\mu}-v \cdot q v_{\mu} . I=\sqrt{2}, 1$ for charged and neutral pion respectively. It can be shown [7] by combining heavy quark symmetry and chiral symmetry that the constants in (3)-(5) satisfy

$$
g\left(B_{2}^{*}, B\right)=g\left(B_{2}^{*}, B^{*}\right)=\sqrt{\frac{2}{3}} g\left(B_{1}, B^{*}\right) \equiv \mathcal{G} .
$$

For deriving the sum rules for the constants in the decay amplitudes (5), (4) and (5) we consider the correlators 


$$
\begin{gathered}
\int d^{4} x e^{-i k \cdot x}\langle\pi(q)| T \\
\left(J_{1,-, \frac{1}{2}}^{\beta}(0) J_{1,+, \frac{3}{2}}^{\dagger \alpha}(x)\right)|0\rangle \\
=\iint d^{4} x d^{4} y e^{-i k \cdot x+i k^{\prime} \cdot y}\left\langle 0\left|T\left(J_{1,-, \frac{1}{2}}^{\beta}(y) \frac{1}{f_{\pi}} \partial^{\mu} j_{\mu}^{5}(0) J_{1,+, \frac{3}{2}}^{\dagger \alpha}(x)\right)\right| 0\right\rangle \\
=\left(q_{t}^{\alpha} q_{t}^{\beta}-\frac{1}{3} g_{t}^{\alpha \beta} q_{t}^{2}\right) G_{B_{1} B^{*}}\left(\omega, \omega^{\prime}\right) \\
\int d^{4} x e^{-i k \cdot x}\left\langle\pi(q)\left|T\left(J_{0,-, \frac{1}{2}}(0) J_{2,+, \frac{3}{2}}^{\dagger \alpha \beta}(x)\right)\right| 0\right\rangle=\left(q_{t}^{\alpha} q_{t}^{\beta}-\frac{1}{3} g_{t}^{\alpha \beta} q_{t}^{2}\right) G_{B_{2}^{*} B}\left(\omega, \omega^{\prime}\right), \\
\int d^{4} x e^{-i k \cdot x}\left\langle\pi(q)\left|T\left(J_{1,-, \frac{1}{2}}^{\gamma}(0) J_{2,+, \frac{3}{2}}^{\dagger \alpha \beta}(x)\right)\right| 0\right\rangle=\frac{i}{2}\left(\varepsilon^{\rho \gamma \alpha \sigma} v_{\rho} q_{t \sigma} q_{t}^{\beta}+\alpha \leftrightarrow \beta\right) G_{B_{2}^{*} B^{*}}\left(\omega, \omega^{\prime}\right),
\end{gathered}
$$

where $k=k^{\prime}+q, \omega=2 v \cdot k, \omega^{\prime}=2 v \cdot k^{\prime}$ and $q^{2}=0$. PCAC is used in the first equality of (7). The forms of the right hand side of (7), (8) and (9) are determined by that $\alpha, \beta$ and $\gamma$ are transverse indices, $x-y=v t$ on the heavy quark propagator and that the pion is in the $D$ wave state due to conservation of angular momentum of the light component. The interpolationg currents are given in [5] as

$$
\begin{aligned}
& J_{1,+, \frac{3}{2}}^{\dagger \alpha}=\sqrt{\frac{3}{4}} \bar{h}_{v} \gamma^{5}(-i)\left(\mathcal{D}_{t}^{\alpha}-\frac{1}{3} \gamma_{t}^{\alpha} \mathcal{D}_{t}\right) q, \\
& J_{2,+, \frac{3}{2}}^{\dagger \alpha_{1}, \alpha_{2}}=\sqrt{\frac{1}{2}} \bar{h}_{v} \frac{(-i)}{2}\left(\gamma_{t}^{\alpha_{1}} \mathcal{D}_{t}^{\alpha_{2}}+\gamma_{t}^{\alpha_{2}} \mathcal{D}_{t}^{\alpha_{1}}-\frac{2}{3} g_{t}^{\alpha_{1} \alpha_{2}} \mathcal{D}_{t}\right) q, \\
& J_{1,-, \frac{1}{2}}^{\dagger \alpha}=\sqrt{\frac{1}{2}} \bar{h}_{v} \gamma_{t}^{\alpha} q, \quad J_{0,-\frac{1}{2}}^{\dagger \alpha}=\sqrt{\frac{1}{2}} \bar{h}_{v} \gamma_{5} q,
\end{aligned}
$$

where $h_{v}$ is the heavy quark field in HQET and $\gamma_{t \mu}=\gamma_{\mu}-v_{\mu} \psi$.

Let us first consider the function $G_{B_{1} B^{*}}\left(\omega, \omega^{\prime}\right)$ in (7). As a function of two variables, it has the following pole terms from double dispersion relation

$$
\frac{f_{-, \frac{1}{2}} f_{+, \frac{3}{2}} g\left(B_{1} B^{*}\right)}{\left(2 \bar{\Lambda}_{-, \frac{1}{2}}-\omega^{\prime}\right)\left(2 \bar{\Lambda}_{+, \frac{3}{2}}-\omega\right)}+\frac{c}{2 \bar{\Lambda}_{-, \frac{1}{2}}-\omega^{\prime}}+\frac{c^{\prime}}{2 \bar{\Lambda}_{+, \frac{3}{2}}-\omega},
$$

where $f_{P, j_{\ell}}$ are constants defined in (11), $\bar{\Lambda}_{P, j_{\ell}}=m_{P, j_{\ell}}-m_{Q}$. As explained in Section 四, only one state with $j^{P}=1^{+}$contributes to (13) as the result of equation (11). This would not be true if the last term in (10) is absent.

Usually, the soft-pion approximation $\omega=\omega^{\prime}$ is used in QCD sum rules for decay amplitudes. This is not a good approximation since the difference $\omega-\omega^{\prime}=2 v \cdot q$ is about $1 \mathrm{GeV}$ in the real decay process as can be seen from the observed spectra of $D$ system and the calculation results of [5]. We instead put 


$$
\omega-\omega^{\prime}=2\left(\bar{\Lambda}_{+, \frac{3}{2}}-\bar{\Lambda}_{-, \frac{1}{2}}\right)=2 \Delta .
$$

This is satisfied by the real decay process in the leading order of $1 / m_{Q}$. We then use dispersion relation of one variable to $G_{B_{1} B^{*}}\left(\omega, \omega^{\prime}\right)$, the pole terms of which are

$$
\frac{f_{-, \frac{1}{2}} f_{+, \frac{3}{2}} g\left(B_{1} B^{*}\right)}{\left(2 \bar{\Lambda}_{-, \frac{1}{2}}-\omega^{\prime}\right)^{2}}+\frac{c+c^{\prime}}{\left(2 \bar{\Lambda}_{-, \frac{1}{2}}-\omega^{\prime}\right)} .
$$

The free heavy quark propagator in HQET is $\int d \tau \delta(x-v \tau) \frac{1+\psi}{2}$. The interaction of the heavy quark with the gluon field $A$ in the leading order of $1 / m_{Q}$ expansion is $g \bar{h} v \cdot A h$. We shall use the Fock-Schwinger gauge $x_{\mu} A^{\mu}(x)=0$ throughout this paper. In this gauge the full propagator of the heavy quark $\langle 0|T(h(0) \bar{h}(x))| 0\rangle$ in the leading order of $1 / m_{Q}$ is identical to the free one, because $v=x / \tau$ on the heavy quark line. Therfore, the correlator (7) has the form

$$
\int d \tau e^{i \omega \tau} \operatorname{tr}\left\{\Gamma\left\langle\pi(q)\left|D_{t} q(v \tau) \bar{q}(0)\right| 0\right\rangle\right\}=\left(q_{t}{ }^{\alpha} q_{t}{ }^{\beta}-g_{t}^{\alpha \beta}\right) \int d \tau e^{i \omega \tau} f(v q, \tau),
$$

where $\Gamma$ is a vertex factor containing $\gamma$ matrices. Since in our approach $v \cdot q$ is fixed to be equal to $\Delta$ by (114) and the invaraint function $G\left(B_{1} B^{*}\right)$ is considered as a function of a single variable $\omega$ (or $\left.\omega^{\prime}\right)$, the integral in $\tau$ is dominated by small $\tau$, that is, the small distance $x=v \cdot \tau$ for $\omega$ in the deep euclidian region. Therefore for deriving QCD sum rules we can calculate the correlator (7) by the operator product expansion. This is an expansion over the small quantity $\tau$, whch after the integration becomes an assymptotic expansion in powers of $1 / \omega$. By dimensional reason it is a power series in $v \cdot q / \omega$ and $\Lambda_{Q C D} / \omega$ which is meaningful for sufficiently large value of $\omega$. After Borel transform the series is convergent when $v \cdot q / T$ is sufficiently small, where $\mathrm{T}$ is the Borel transform parameter. We emphasize that this is so because $v \cdot q$ is fixed in our approach. The perturbation terms do not contribute since they do not violate the conservation equation $\partial^{\mu} j_{\mu}^{5}=0$. For the higher terms we expand the quark field $q(x)$ in the matrix element $\langle\pi(q)|T(q(x) \bar{q}(0))| 0\rangle$. The matrix elements appearing in the expansion have the general form (37), (38) and (39) in Appendix. The terms in (37)-(39) which contribute to the QCD sum rules in the soft 
pion approximation were used in [4]. Here we keep all terms in these formulas in the calculations in order to avoid the soft pion approximation. Substituting these equations in the correlator (7) and making Borel transform to supress the contribution of higher states we find the sum rule in the leading order of HQET.

$$
\begin{aligned}
f_{-, \frac{1}{2}} f_{+, \frac{3}{2}} \frac{g\left(B_{1} B^{*}\right)}{T} e^{-2 \bar{\Lambda}_{-, \frac{1}{2}} / T} & +\left(c+c^{\prime}\right) e^{-2 \bar{\Lambda}_{-, \frac{1}{2}} / T}+\cdots= \\
& \sqrt{\frac{3}{2}}\left[g_{2}+\frac{2}{T}\left(c_{2}-d_{2}-e_{2} \Delta\right)\right] e^{2 \Delta / T} .
\end{aligned}
$$

In the above equation we have neglected all radiative corrections and operators with dimension $\geq 6$. In the derivation of above equation we need to rotate the integration path clock-wise to the imaginary axis of $\tau$ and redefine the integral variable $\tau^{\prime}=i \tau$. The result is then obtained by using the Borel transform formula $\hat{B}_{T}^{\omega} \int d \tau e^{i \tau \omega} \tau^{n}=(-i)^{n+1}(1 / T)^{n}$ in the negative region of $\omega$.

The dots in the left hand side of (17) represents terms from the contributions from the radial excited states of the same $j^{P}$ and $j_{\ell}$ and the continuum. In order to estimate these terms we make the duality assumption that above $\omega=\omega_{c}$ the dispersion integral of the hadron side is equal to that obtained from the QCD calculation. The spectra function $\rho_{\mathrm{QCD}}(\omega)$ of the latter is obtained by performing the Borel transform $\hat{B}_{1 / \omega}^{T}$ to the right hand side of (17). The result for $\rho_{\mathrm{QCD}}(\omega)$ contains two terms proportional to $\delta(\omega-2 \Delta)$ and $\delta^{\prime}(\omega-2 \Delta)$ respectively. This implies that $\int_{\omega_{c}}^{\infty} \frac{\rho_{\mathrm{QCD}}\left(\omega^{\prime}\right) d \omega^{\prime}}{\omega^{\prime}-\omega}$ vanishes for $\omega_{c}>2 \Delta \sim 1$ $\mathrm{GeV}$. Therefore we shall neglect the contributions from the radial excited states and the continuum to the sum rule (17).

We can multiply (17) by $\exp \left(2 \bar{\Lambda}_{-, \frac{1}{2}} / T\right)$ and differentiate in $1 / T$ in order to eliminate $c$ and $c^{\prime}$. By using (6) we obtain the following sum rule for the constant $\mathcal{G}$

$$
\mathcal{G} f_{-, \frac{1}{2}} f_{+, \frac{3}{2}}=2\left\{\bar{\Lambda}_{+, \frac{3}{2}} g_{2}+\left(c_{2}-d_{2}-e_{2} \Delta\right)\left(1+\frac{2 \bar{\Lambda}_{+, \frac{3}{2}}}{T}\right)\right\} e^{2 \bar{\Lambda}_{+, \frac{3}{2}} / T}
$$

The sum rules for the correlators (8) and (9) can be obtained by similar calculations. Equations similar to (18) are obtained and we have checked that the relations in ( 0 ) are satisfied. 
The decay amplitudes of the members of the doublet $(j,+, 1 / 2), B_{0}^{\prime}$ and $B_{1}^{\prime}$, have the following form

$$
\begin{aligned}
M\left(B_{0}^{\prime} \rightarrow B \pi\right) & =I \mathcal{G}^{\prime} \\
M\left(B_{1}^{\prime} \rightarrow B^{*} \pi\right) & =I \epsilon^{*} \cdot \eta \mathcal{G}^{\prime}
\end{aligned}
$$

in the $m_{Q} \rightarrow \infty$ limit. The sum rule in HQET for the coupling constant $\mathcal{G}^{\prime}$ can be derived similarly. From [5], the interpolating currents for $B_{0}^{\prime}, B_{1}^{\prime}$ can be chosen as

$$
J_{0,+, \frac{1}{2}}^{\dagger}=\frac{1}{\sqrt{2}} \bar{h}_{v} q, \quad J_{1,+, \frac{1}{2}}^{\dagger \alpha}=\frac{1}{\sqrt{2}} \bar{h}_{v} \gamma^{5} \gamma_{t}^{\alpha} q
$$

As pointed out in [5], for any state $\left(j, P, j_{\ell}\right)$ besides the interpolating current with the lowest dimension there is another one which has an additional factor $-i \mathcal{D}_{t}$ inserting before $q$. For the doublet $(j,+, 1 / 2)$, they are

$$
J_{0,+, \frac{1}{2}}^{\dagger \dagger}=\frac{1}{\sqrt{2}} \bar{h}_{v}(-i) \mathcal{D}_{t} q, \quad J_{1,+, \frac{1}{2}}^{\dagger}=\frac{1}{\sqrt{2}} \bar{h}_{v} \gamma^{5} \gamma_{t}^{\alpha}(-i) \mathcal{D}_{t} q
$$

As discussed in [5], there is some motivation for using the latter currents for the corresponding constant $f_{+, 1 / 2}^{\prime}$ survives in the non-relativistic limit of the Bethe-Salpeter wave function, while the constant $f_{+, 1 / 2}$ for the current (21) vanishes in this limit, since $B_{0}^{\prime}$ and $B_{1}^{\prime}$ are $P$ wave states in the non-relativistic quark model.

After Borel transformation and eliminating the single pole terms by differentiation, the sum rule derived by using the interpolating current (22) is

$$
\begin{aligned}
f_{-, \frac{1}{2}} f_{+, \frac{1}{2}}^{\prime} \mathcal{G}^{\prime}= & 2\left[\bar{\Lambda}_{+, \frac{1}{2}}\left(-g_{2} \Delta^{2}+3 h_{2} \Delta+3 h_{1}\right)-\left(-3 b_{1}+3 c_{1} \Delta+3 d_{1} \Delta\right.\right. \\
& \left.\left.+4 c_{2} \Delta^{2}+2 d_{2} \Delta^{2}-e_{2} \Delta^{3}\right)\left(1+\frac{2 \bar{\Lambda}_{+, \frac{1}{2}}}{T}\right)\right] e^{2 \bar{\Lambda}_{+, \frac{1}{2}} / T}
\end{aligned}
$$

where $\Delta=\bar{\Lambda}_{+, 1 / 2}^{\prime}-\bar{\Lambda}_{-, 1 / 2}$. If the interpolating current (21) is used instead of (22), the sum rule turns out to be

$$
f_{-, \frac{1}{2}} f_{+, \frac{1}{2}} \mathcal{G}^{\prime}=\left(\left(-\bar{\Lambda}_{+, \frac{1}{2}} \alpha-\frac{\beta}{2}\right)-\frac{\bar{\Lambda}_{+, \frac{1}{2}} \beta-\gamma}{T}+\frac{\bar{\Lambda}_{+, \frac{1}{2}} \gamma}{T^{2}}\right) e^{2 \bar{\Lambda}_{+, \frac{1}{2}} / T}
$$

where 


$$
\begin{aligned}
& \alpha=2\left(f_{1}+f_{2} \Delta\right), \quad \beta=4\left(g_{1} \Delta+g_{2} \Delta^{2}+h_{1}\right), \\
& \gamma=4\left(a_{1}+a_{2} \Delta+2 c_{1} \Delta+e_{1} \Delta^{2}+e_{2} \Delta^{3}\right)
\end{aligned}
$$

and $\Delta=\bar{\Lambda}_{+, 1 / 2}-\bar{\Lambda}_{-, 1 / 2}$.

\section{DETERMINATION OF THE PARAMETERS}

In order to obtain the decay constants $\mathcal{G}$ and $\mathcal{G}^{\prime}$ from (18), (23) and (24) we need to use the mass parameters $\bar{\Lambda}$ 's and the coupling constants f's of the corresponding interpolating currents as input. $\bar{\Lambda}_{-, 1 / 2}$ and $f_{-, 1 / 2}$ can be obtained from the results in $[8]$ as $\bar{\Lambda}_{-, 1 / 2}=0.5$ $\mathrm{GeV}$ and $f_{-, 1 / 2} \simeq 0.24 \mathrm{GeV}^{3 / 2}$ at the order $\alpha_{s}=0$. Notice that the coupling constant $f_{-, 1 / 2}$

defined in the present work is a factor $1 / \sqrt{2}$ smaller than that defined in $[\mathbb{B}] \cdot \bar{\Lambda}_{+, 3 / 2}, \bar{\Lambda}_{+, 1 / 2}$ and $\bar{\Lambda}_{+, 1 / 2}^{\prime}$ are given in [5]. $f_{+, 3 / 2}, f_{+, 1 / 2}$ and $f_{+, 1 / 2}^{\prime}$ can be determined from the formulas $(34),(27)$ and (28) of reference [5] derived from sum rules for two point correlators. The results are

$$
\begin{array}{ll}
\bar{\Lambda}_{+, 3 / 2}=0.95 \mathrm{GeV} & f_{+, 3 / 2}=0.19 \pm 0.03 \mathrm{GeV}^{5 / 2}, \\
\bar{\Lambda}_{+, 1 / 2}^{\prime}=0.9 \mathrm{GeV} & f_{+, 1 / 2}^{\prime}=0.28 \pm 0.03 \mathrm{GeV}^{5 / 2}, \\
\bar{\Lambda}_{+, 1 / 2}=1.05 \mathrm{GeV} & f_{+, 1 / 2}=0.32 \pm 0.03 \mathrm{GeV}^{3 / 2} .
\end{array}
$$

(40)-(45) still contains several parameters. $m_{0}^{2}$ is known to be $0.8 \mathrm{GeV}^{2}$. $m_{1}^{2}$ was calculated in [9] with QCD sum rules with the result $m_{1}^{2}=0.2 \mathrm{GeV}^{2}$. We have obtained QCD sum rules for $e_{1}$ and $e_{2}$ by considering the two point functions

$$
\begin{aligned}
& i \int e^{i q \cdot x} d^{4} x\langle 0| T\left\{\bar{q}(x) i \gamma_{5} \frac{\tau^{a}}{2} q(x) \bar{q}(0) \frac{\tau^{b}}{2}(n \cdot D)^{2} i \gamma_{5} q(0)|0\rangle=(n \cdot q)^{2} A\left(q^{2}\right) \delta_{a b},\right. \\
& i \int e^{i q \cdot x} d^{4} x\langle 0| T\left\{\bar{q}(x) \not h \gamma_{5} \frac{\tau^{a}}{2} q(x) \bar{q}(0) \frac{\tau^{b}}{2}(n \cdot D)^{2} \not h \gamma_{5} q(0)|0\rangle=(n \cdot q)^{4} B\left(q^{2}\right) \delta_{a b}\right.
\end{aligned}
$$

where $n^{2}=0$. The resulting QCD sum rules are

$$
\frac{2 e_{1}}{f_{\pi}}\langle\bar{q} q\rangle_{0}=\frac{1}{16 \pi^{2}} \int_{0}^{s_{0}} e^{-s / M^{2}} d s+\frac{1}{24 \pi}\left\langle\alpha_{s} G^{2}\right\rangle_{0}+\frac{112}{81} \pi \alpha_{s} \frac{\langle\bar{q} q\rangle_{0}^{2}}{M^{2}}
$$




$$
\begin{aligned}
2 e_{2} f_{\pi} m_{A}^{2}= & \frac{3}{80 \pi^{2}} \int_{0}^{s_{0}}\left(m_{A}^{2}-s\right) e^{-s / M^{2}} d s+\frac{1}{48 \pi}\left\langle\alpha_{s} G^{2}\right\rangle_{0}\left(1+\frac{m_{A}^{2}}{M^{2}}\right) \\
& +\frac{20}{27} \pi \alpha_{s}\left(2+\frac{m_{A}^{2}}{M^{2}}\right) \frac{\langle\bar{q} q\rangle_{0}^{2}}{M^{2}} .
\end{aligned}
$$

In obtaining (30) we have eliminated the $a_{1}$ pole term by differentiation with respect to the Borel parameter $1 / M^{2} \cdot m_{A} \simeq 1.3 \mathrm{GeV}$ is the mass of $a_{1}$. In the numerical calculations for $e_{1}$ and $e_{2}$ we use the following standard values of the vacuum condensates:

$$
\begin{aligned}
\langle\bar{q} q\rangle_{0} & =-(0.24 \mathrm{GeV})^{3}, \\
\left\langle\alpha_{s} G G\right\rangle_{0} & =0.038 \mathrm{GeV}^{4}
\end{aligned}
$$

as well as $\alpha_{s}=0.4$. From (29) and (30) we obtain the following numerical values for $e_{1}$ and $e_{2}$,

$$
\begin{aligned}
& e_{1}=-0.014 \pm 0.002 \quad \mathrm{GeV}^{2} \\
& e_{2}=0.015 \pm 0.002 \mathrm{GeV}
\end{aligned}
$$

\section{NUMERICAL RESULTS AND DISCUSSION}

We now turn to the numerical evaluation of the sum rules for $\mathcal{G}$ and $\mathcal{G}^{\prime}$. By using (26) and (31) together with the values of parameters determined above we obtain $\mathcal{G}$ and $\mathcal{G}^{\prime}$ as functions of $T$ from the sum rules (18), (23) and (24). The results are plotted as curves in fig. 1. The lower limit of $T$ is determined by the requirement that in the sum rules before differentiating with respect to $1 / T$ ( such as (17)), the terms of higher order in $1 / T$ in the operator expansion are reasonably smaller than the leading term, say $\leq 1 / 3$ of the latter. This leads to $T>1.2 \mathrm{GeV}$ and $T>3 \mathrm{GeV}$ for the sum rule (18) and (23) respectively.

Usually the value of $T$ is constrained from the upper side by the requirement that the contribution from higher states is sufficiently suppressed. The continuum model discussed below equation (17) allows $T$ to be any large value. However, in order that the results depend less on the continuum model it is good if $T$ is not much larger than the $2\left(\bar{\Lambda}^{(1)}-\bar{\Lambda}\right)$, 
where $m_{Q}+\bar{\Lambda}^{(1)}$ is the mass of the radial excited state. Quark model calculations 10 indicate that $\bar{\Lambda}^{(1)}-\bar{\Lambda}$ is roughly $0.7 \mathrm{GeV}$. Therefore, this requirement can be satisfied for the sum rule for $\mathcal{G}$. We find within the stability window subject to the constrain disussed above

$$
\mathcal{G}=5.7 \pm 0.6 \pm 0.7 \quad \mathrm{GeV}^{-2}, \quad 1.3 \mathrm{GeV}<T<1.5 \mathrm{GeV}
$$

From the view point stated above, the sum rule (23) for $\mathcal{G}^{\prime}$ is not very satisfactory since the lower limit of $T$ is too high. If we somewhat arbitrarily take the width of the window to be $0.3 \mathrm{GeV}$, we find from (23)

$$
\mathcal{G}^{\prime}=1.0 \pm 0.1 \pm 0.5, \quad 3 \mathrm{GeV}<T<3.3 \mathrm{GeV}
$$

In (33) and (34) the first error refers to the variation with $T$ in this region, while the second error takes into account the uncertainty in the values of the coupling constant $f$ 's. The errors quoted above do not include those from higher order terms in OPE and the $1 / m_{Q}$ expansion and the values of parameters characterizing the condensations.

However,the (33) and (34) have another source of uncertainty. These sum rules depend on the parameter $e_{1}$ which is obtained by the sum rule (29). It is well known [9] that the sum rules derived from the correlator of two pseudo-scalar currents give too small value for the physical quantities. In the case of two pseudo-scalar current without derivative, the phenomenogical side is about a factor 2 larger than the theoretical side. Just for a rough estimation, let us multiplying the $e_{1}$ value by a factor 2 . We find that the $T$ value at which the last term is $1 / 3$ of the first term in the sum rule before differention with respect to $1 / T$ move to lower values $0.6 \mathrm{GeV}$ and $1.9 \mathrm{GeV}$ for the sum rule (17) and (23) respectively. That is, the convergence becomes faster. The values of $\mathcal{G}$ and $\mathcal{G}^{\prime}$ change to

$$
\begin{array}{ll}
\mathcal{G}=3.6 \pm 1.0 \pm 0.7 \mathrm{GeV}^{-2}, & 1.0 \mathrm{GeV}<T<1.5 \mathrm{GeV} \\
\mathcal{G}^{\prime}=1.2 \pm 0.1 \pm 0.5, & 1.8 \mathrm{GeV}<T<2.1 \mathrm{GeV}
\end{array}
$$

On the other hand the sum rule (24) is not good in stability. Especially, $\mathcal{G}^{\prime}$ changes sign near $T \simeq 1.2 \mathrm{GeV}$. Therefore, we do not use it in the numerical calculation of $\mathcal{G}^{\prime}$. 
The decay widths in the leading order are

$$
\begin{aligned}
& \Gamma\left(B_{1} \rightarrow B^{*} \pi\right)=\frac{1}{8 \pi} \mathcal{G}^{2}|\vec{q}|^{5}, \\
& \Gamma\left(B_{2}^{*} \rightarrow B \pi\right)=\frac{1}{20 \pi} \mathcal{G}^{2}|\vec{q}|^{5}, \\
& \Gamma\left(B_{2}^{*} \rightarrow B^{*} \pi\right)=\frac{3}{40 \pi} \mathcal{G}^{2}|\vec{q}|^{5}, \\
& \Gamma\left(B_{0}^{\prime} \rightarrow B \pi\right)=\frac{3}{8 \pi} \mathcal{G}^{\prime 2}|\vec{q}|, \\
& \Gamma\left(B_{1}^{\prime} \rightarrow B^{*} \pi\right)=\frac{3}{8 \pi} \mathcal{G}^{\prime 2}|\vec{q}|,
\end{aligned}
$$

where sum over charged and neutral pion final states has been included in the above formulas.

In the following we apply the leading order formulas obtained above to the excited states of charmed mesons. The value $\vec{q}$ for the first three processes in (36) are calculated from the experimental mass values of the relevent particles [11] and that for the last two processes are calculated from the difference between the theoretical mass values of the two doublets in the leading order obtained in [5] and [8]. For the excited states of $D$ we obtain the results listed in table \, where the numbers in the parentheses are results for $e_{1}=-0.028$. The experimental data [1] and the results of [3] obtained with relativistic B-S equation are also included. For the $D$ series the $\mathcal{O}\left(1 / m_{Q}\right)$ corrections are not negligible. Yet, assuming the total width of $D_{2}^{*}$ is dominated by one pion decay, the calculation result for $D_{2}^{*}$ is in reasonable agreement with experimental data. The calculation result for $\Gamma\left(D_{1} \rightarrow D^{*} \pi\right)$ in the leading order is significantly smaller than the experimental data for $\Gamma\left(D_{1} \rightarrow\right.$ all $)$. This can partly be attributed to mixing of the two $1^{+}$states in the order of $\mathcal{O}\left(1 / m_{Q}\right)$ [7]. This effect can be calculated within our formalism by introducing the $\mathcal{O}\left(1 / m_{Q}\right)$ terms in the Largrangian of HQET. In reference [1]], it was estimated that the $\Gamma\left(D_{1} \rightarrow D \rho+D^{*} \rho\right) \simeq 6$ $\mathrm{MeV}, \Gamma\left(D_{2}^{*} \rightarrow D \rho+D^{*} \rho\right) \simeq 3 \mathrm{MeV}$. Therefore, the effect of mixing of two $1^{+}$states in the order $\mathcal{O}\left(1 / m_{Q}\right)$ needs not to be very large. The calculated results for the decay widths of $D_{0}^{\prime}$ and $D_{1}^{\prime}$ in the leading order is too small. This may due to the slow convergence of Eq. (23). 


\section{ACKNOWLEDGMENTS}

This work was supported in part by the National Natural Science Foundation of China.

One of the authers (C.L.) was supported in part by the KOSEF through the SRC programm.

Appendix: The OPE of matrix element $\left\langle\pi^{i}(q)|T(q(x) \bar{q}(0))| 0\right\rangle$

$$
\begin{aligned}
& \left\langle\pi^{i}(q)\left|q_{\alpha}^{a}(0) \bar{q}_{\beta}^{b}(0)\right| 0\right\rangle=i\left(f_{1} \gamma_{5}+f_{2} \not q \gamma_{5}\right)_{\alpha \beta}\left(\frac{\tau_{i}}{2}\right)_{a b}, \\
& \left\langle\pi^{i}(q)\left|D_{\mu} q_{\alpha}^{a}(0) \bar{q}_{\beta}^{b}(0)\right| 0\right\rangle=\left\{\left(g_{1}+g_{2} \not\right) q_{\mu} \gamma_{5}+h_{1} \gamma_{\mu} \gamma_{5}+h_{2} i \sigma_{\nu \mu} \gamma_{5} q^{\nu}\right\}_{\alpha \beta}\left(\frac{\tau_{i}}{2}\right)_{a b}, \\
& \left\langle\pi^{i}(q)\left|D_{\mu} D_{\nu} q_{\alpha}^{a}(0) \bar{q}_{\beta}^{b}(0)\right| 0\right\rangle=i\left\{\left[g_{\mu \nu}\left(a_{1}+a_{2} \not\right)-i \sigma_{\mu \nu} b_{1}+i \varepsilon_{\mu \nu \rho \sigma} \gamma^{\rho} q^{\sigma} \gamma_{5} b_{2}\right.\right. \\
& +\left(q_{\mu} \gamma_{\nu}+q_{\nu} \gamma_{\mu}\right) c_{1}+\left(q_{\mu} \gamma_{\nu}-q_{\nu} \gamma_{\mu}\right) d_{1} \\
& +i\left(q_{\mu} \sigma_{\lambda \nu}+q_{\nu} \sigma_{\lambda \mu}\right) q^{\lambda} c_{2}+i\left(q_{\mu} \sigma_{\lambda \nu}-q_{\nu} \sigma_{\lambda \mu}\right) q^{\lambda} d_{2} \\
& \left.\left.+\left(e_{1}+e_{2} \not\right) q_{\mu} q_{\nu}\right] \gamma_{5}\right\}_{\alpha \beta}\left(\frac{\tau_{i}}{2}\right)_{a b} \text {; } \\
& f_{1}=-\frac{1}{2 f_{\pi}}\langle\bar{q} q\rangle_{0}, \quad f_{2}=-\frac{f_{\pi}}{2} \\
& g_{1}=\frac{1}{4 f_{\pi}}\langle\bar{q} q\rangle_{0}, \quad g_{2}=\frac{f_{\pi}}{4}, \quad h_{1}=0, \quad h_{2}=-\frac{1}{12 f_{\pi}}\langle\bar{q} q\rangle_{0} \text {; } \\
& a_{1}=-\frac{m_{0}^{2}}{16 f_{\pi}}\langle\bar{q} q\rangle_{0}, \quad a_{2}=-\frac{5}{36} f_{\pi} m_{1}^{2}, \quad c_{1}=\frac{1}{36} f_{\pi} m_{1}^{2}, \quad c_{2}=-\frac{1}{24 f_{\pi}}\langle\bar{q} q\rangle_{0}
\end{aligned}
$$

where $\left\langle\pi^{i}(q)\left|\bar{q} \frac{\tau_{i}}{2} D^{2} \gamma_{\rho} \gamma_{5} q\right| 0\right\rangle=-i f_{\pi} m_{1}^{2} q_{\rho}$.

$$
\begin{aligned}
& e_{1}(n \cdot q)^{2}=\frac{i}{2}\left\langle\pi^{i}(q)\left|\bar{q} \frac{\tau_{i}}{2}(n \cdot D)^{2} \gamma_{5} q\right| 0\right\rangle, \quad \text { for } \quad n^{2}=0, \\
& e_{2}(n \cdot q)^{3}=\frac{i}{2}\left\langle\pi^{i}(q)\left|\bar{q} \frac{\tau_{i}}{2} \gamma_{5} \not h(n \cdot D)^{2} q\right| 0\right\rangle, \quad \text { for } \quad n^{2}=0, \\
& b_{1}=\frac{a_{1}}{3}, \quad b_{2}=-3 c_{1}, \quad d_{1}=0, \quad d_{2}=-\frac{e_{1}}{2}+\frac{1}{12 f_{\pi}}\langle\bar{q} q\rangle_{0} .
\end{aligned}
$$

Here $f_{\pi}=93 \mathrm{MeV}$. 


\section{REFERENCES}

[1] E. Eichten, C. T. Hill and C. Quigg, Phys. Rev. lett. 71, 4116(1993); Fermilab-Conf94/118-T (1994).

[2] Y. B. Dai, C. S. Huang and H. Y. Jin, Phys. Lett. B331, 174(1994).

[3] Y. B. Dai and H. Y. Jin, Phys. Rev. D 52, 236(1995).

[4] P. Colangelo, G. Nardulli, A. A. Ovchinnikov and N. Paver, Phys. Lett. B269, 204(1991); P. Colangelo, G. Nardulli and N. Paver, Phys. Lett. B293, 207(1992); P. Colangelo, F. De Fazio, G. Nardulli, N. Di Bartolomeo and R. Gatto, Phys. Rev. D 52, 6422(1995).

[5] Y. B. Dai, C. S. Huang, M. Q. Huang and C. Liu, Phys. Lett. B390, 350(1997); Y. B. Dai, C. S. Huang and M. Q. Huang, Phys. Rev. D 55, 5719(1997).

[6] B. Grinstein, Nucl. Phys. B339, 253(1990); E. Eichten and B. Hill, Phys. Lett. B234, 511(1990); A. F. Falk, H. Georgi, B. Grinstein and M. B. Wise, Nucl. Phys. B343, 1(1990); F. Hussain, J. G. Körner, K. Schilcher, G. Thompson and Y. L. Wu, Phys. Lett. B249, 295(1990); J. G. Körner and G. Thompson, Phys. Lett. B264, 185(1991).

[7] A. Falk and M. Luke, Phys. Lett. B292, 119(1992); U. Kilian, J.G. Körner, and D. Pirjol, Phys. Lett. B288, 360(1992).

[8] E. Bagen, P. Ball, V. M. Braun and H. G. Dosch, Phys. Lett. B278, 457(1992); M. Neubert, Phys. Rev. D 45, 2451(1992); D. J. Broadhurst and A. G. Grozin, Phys. Lett. B274, 421(1992).

[9] A. V. Smilga and M. A. Shifman, Yad. Fiz. 37, 1613(1983); V. A. Novikov, M. A. Shifman, A. I. Vainshtein, M. B. Voloshin and V. I. Zakharov, Nucl. Phys. B237, $525(1984)$

[10] S. Godfrey and N. Isgur, Phys. Rev. D 32, 189(1985); J. Morishita, M. Kawaguchi 
and T. Morii, Phys. Rev. D 37, 159(1988).

[11] Particle Data Group, R. M. Barnett et. al., Phys. Rev. D 54, 1(1996). 


\section{Figure Captions}

Fig. 1. Dependence of $\mathcal{G}$ and $\mathcal{G}^{\prime}$ on the Borel parameter $T$. The three curves correspond, from top to bottom, to the sum rules (18), (23) and (24), respectively. 


\section{TABLES}

TABLE I. The decay widths of $D_{1}, D_{2}^{*}, D_{1}^{\prime}$ and $D_{0}^{\prime}$. The numbers in the parenthesises are results obtained by using $e_{1}=-0.028$ instead of -0.014 .

\begin{tabular}{|l|ccc|}
\hline \hline Transition & Calculated Width $(\mathrm{MeV})$ & Observed Width(MeV) [1] & Result from [3] \\
\hline$\Gamma\left(D_{1} \rightarrow D^{*} \pi\right)$ & $7.0(2.8)$ & & 8.0 \\
\hline$\Gamma\left(D_{1} \rightarrow\right.$ all $)$ & & $18.9_{-3.5}^{+4.6}$ & \\
\hline$\Gamma\left(D_{2}^{*} \rightarrow D \pi\right)$ & $18.2(7.3)$ & & 6.8 \\
\hline$\Gamma\left(D_{2}^{*} \rightarrow D^{*} \pi\right)$ & $6.9(2.8)$ & $23_{-5}^{+5}$ & \\
\hline$\Gamma\left(D_{2}^{*} \rightarrow\right.$ all $)$ & & & \\
\hline$\Gamma\left(D_{0}^{\prime} \rightarrow D \pi\right)$ & & & \\
$\Gamma\left(D_{1}^{\prime} \rightarrow D^{*} \pi\right)$ & $45(64)$ & & \\
\hline \hline
\end{tabular}




\section{FIGURES}

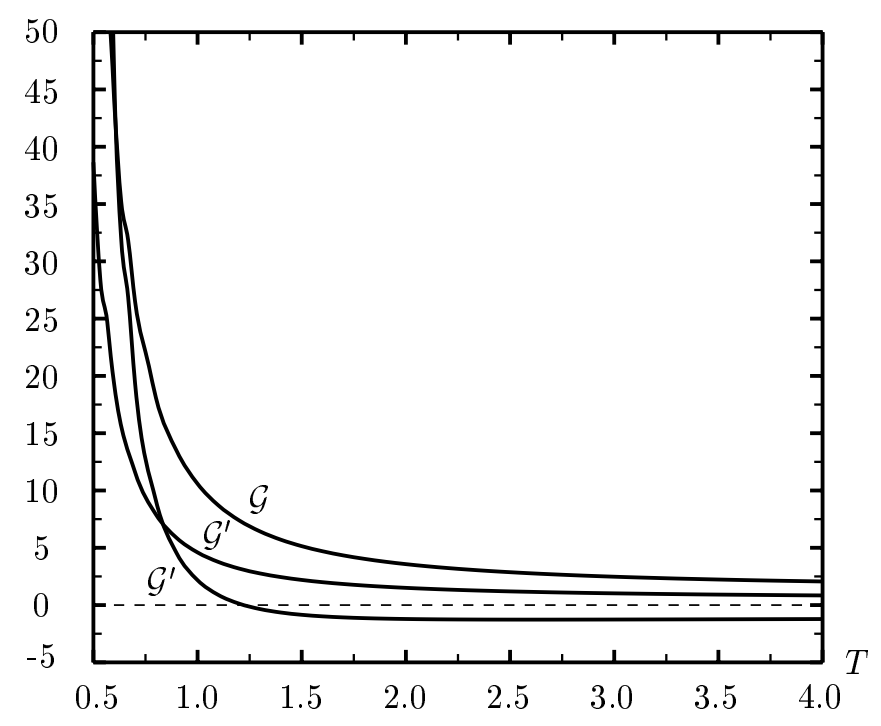

fig. 1 